\title{
Managing Chronic Pain in Osteoarthritis and Rheumatoid Arthritis
}

\author{
Theo Wolf \\ Editorial Assistant \\ Citation: EMJ Rheumatol. 2021;8[1]:25-27.
}

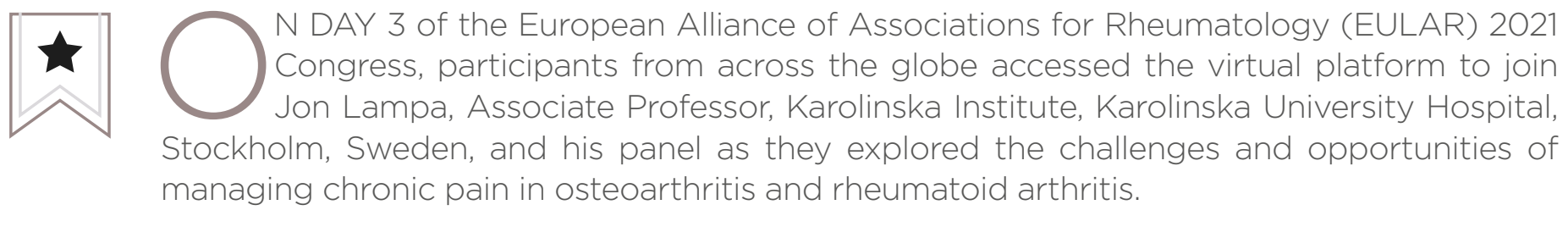

\section{OSTEOARTHRITIS PAIN AND MECHANISM-BASED TREATMENTS}

Malvika Gulati, Rheumatology Registrar, Nuffield Department of Orthopaedics, Rheumatology and Musculoskeletal Sciences, Botnar Research Centre, University of Oxford, UK, began by discussing the management of chronic pain in hand osteoarthritis.

While not required for a diagnosis, blood tests are recommended to exclude alternative causes, such as rheumatoid arthritis or gout. According to Gulati, hand X-rays are often performed to help differentiate hand osteoarthritis from conditions with similar features, including psoriatic arthritis. Common findings from radiographic imaging include loss of joint space, osteophytes, and subchondral sclerosis.

Gulati explained that no single test on its own can be used to define hand osteoarthritis (likelihood ratio <10); however, a composite of features substantially enhances the probability of diagnosis. The chance of a person having hand osteoarthritis when Heberden's nodes alone were present was 20\%; however, this increased to $88 \%$ when coupled with an age over 40 years, a positive family history of nodes, and supportive X-ray features (joint space narrowing in any finger joint).

Pharmacological therapies are frequently administered to individuals with hand osteoarthritis; however, there are numerous other management strategies that could be used, as outlined by Gulati. Education and training in ergonomic principles, the pacing of activities, and the use of assistive devices should be offered to all patients. Moreover, hand exercises can have beneficial effects on pain, function, stiffness, and grip strength. Gulati stressed that patients should be advised to continue with exercises and not view these as a one-off intervention. Orthoses should also be considered for symptom relief in people with thumb-base osteoarthritis. Although a number of patients have reported finding heat helpful, the evidence for a possible beneficial effect is weak and conflicting. With respect to pharmacotherapy, Gulati noted that there is 


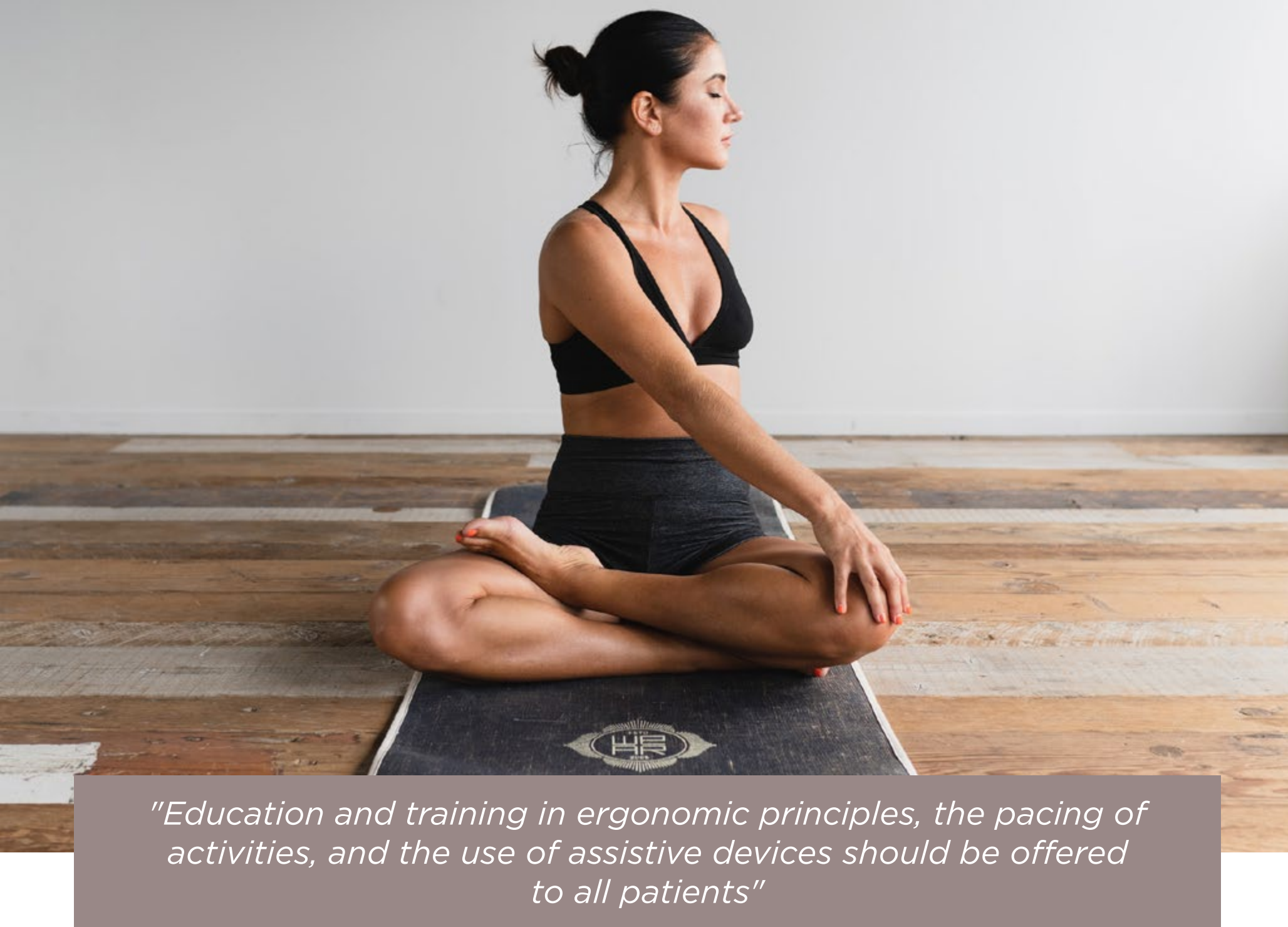

good evidence to support the use of topical antiinflammatories, which are well-tolerated and have a much better side effect profile compared to oral anti-inflammatories. It is recommended that oral analgesics are considered for a limited duration and at the lowest dose possible. There is some evidence for the superiority of chondroitin over placebo for pain and function. Therefore, if patients are keen to take this, they may do so. Trials investigating conventional and biologic disease-modifying anti-rheumatic drugs (e.g., hydroxychloroquine and anti-IL-1) have demonstrated a lack of efficacy. Finally, Gulati mentioned the HOPE study in the Netherlands, in which $10 \mathrm{mg}$ prednisolone was administered for 6 weeks to individuals with inflammatory features of hand osteoarthritis on ultrasound. Although benefit was seen without an excess of adverse events, $10 \mathrm{mg}$ of oral prednisolone is not an insignificant dose. Consequently, Gulati emphasised that glucocorticoids should not be prescribed for prolonged periods of time. Surgery is an option for individuals who have tried the above therapies but continue to be symptomatic. A trapeziectomy should be considered in people with thumb-base osteoarthritis, and arthrodesis or arthroplasty in people with interphalangeal osteoarthritis.

Gulati provided an overview of recent American College of Rheumatology (ACR) recommendations, which advocate the use of cognitive behavioural therapy and centrally acting drugs (e.g., duloxetine). Intra-articular hyaluronic acid is conditionally advised against because studies with a low risk of bias showed no treatment effect.

To summarise, Gulati reiterated the importance of education and exercise, and stressed that treatment must be multidisciplinary and multimodal, rather than solely focused on drugs. 


\section{CHRONIC PAIN IN RHEUMATOID ARTHRITIS AND STRATIFIED TREATMENT APPROACHES}

Neil Basu, Senior Clinical Lecturer of Rheumatology, University of Glasgow, UK, discussed the importance of stratified targeted treatment strategies for chronic pain in rheumatoid arthritis.

Basu started by explaining that patients who respond well to advanced immunotherapies for the control of peripheral inflammation often continue to report clinically significant levels of pain, with one study showing that a substantial proportion of individuals with complete remission still had a Short Form 36 (SF-36) score of $<40$. The disconnect between improvements in the degree of inflammation and improvements in the severity of symptoms suggests that there is contribution from additional pain mechanisms that are distinct from peripheral inflammation. Central sensitisation, the primary underlying cause of chronic pain in fibromyalgia, may represent one such pathway, Basu highlighted.

This is supported by epidemiological data, which found that fibromyalgia has a prevalence of 13$25 \%$ in people with rheumatoid arthritis versus $1-5 \%$ in the general population. From a biological perspective, quantitative sensory testing also lends credence to central sensitisation. Individuals with rheumatoid arthritis and higher 'fibromyalgianess' were found to have a decreased pain threshold around the tibia and other non-articulated surfaces.

Next, Basu explained that the co-existing central pain mechanism artificially inflates commonly used measures of peripheral inflammatory pain. For example, one study found that individuals with concomitant fibromyalgia had a significantly higher disease activity score (DAS) relative to people with rheumatoid arthritis alone (5.36 and 4.03, respectively; $p<0.001$ ). Since many countries use the DAS score to sanction the administration of anti-inflammatory therapies,
Basu revealed that inappropriate prescribing of biological treatments for pain that is not truly inflammatory in origin typically occurs. Indeed, research from Denmark highlighted that $64 \%$ of people with co-existing fibromyalgia and rheumatoid arthritis received biologics relative to $32 \%$ of individuals with rheumatoid arthritis alone.

Basu therefore suggested that EULAR evidencebased guidelines for primary fibromyalgia should be translated to people with co-existing central pain mechanisms and rheumatoid arthritis. The focus is around education and physical therapy. However, for patients who have not responded to either of these strategies, the guidelines indicate a stratified approach. This includes the use of psychological therapies for people with concomitant depression, pharmacotherapy for patients with severe pain and sleep disturbance, and multimodal rehabilitation programmes for individuals who do not do well despite these interventions. With regard to pharmacological treatments, Basu stated that duloxetine has been recommended for the treatment of chronic pain, particularly more central pain mechanisms. A recent study conducted in Japan revealed that this drug provided benefit in people who had achieved remission of rheumatoid arthritis but still suffered pain. Furthermore, an evaluation of various immunotherapies illustrated that JAK inhibitors (e.g., baricitinib) demonstrated a superior analgesic effect relative to the gold standard of anti-TNFa agents. Basu hypothesised that this difference could be explained by the fact JAK inhibitors are better at inhibiting cytokines such as granulocyte-macrophage colony-stimulating factor, which are known to be important in pain pathways.

To conclude, Basu discussed the assessment of pain in people with rheumatoid arthritis. If there is no evidence of inflammation, it is feasible to persevere with existing immunotherapy, focus on other dimensions, such as structural damage, and look beyond the joints, since pain mechanisms may be centrally driven rather than around the periphery. 